\title{
Effects of higher-order dispersion on photon-pair generation by four-wave mixing
}

\author{
Koefoed, Jacob G.; Christensen, Jesper B.; McKinstrie, Colin J.; Rottwitt, Karsten
}

Published in:

Physical Review A

Link to article, DOI:

10.1103/PhysRevA.99.013844

Publication date:

2019

Document Version

Publisher's PDF, also known as Version of record

Link back to DTU Orbit

Citation (APA):

Koefoed, J. G., Christensen, J. B., McKinstrie, C. J., \& Rottwitt, K. (2019). Effects of higher-order dispersion on photon-pair generation by four-wave mixing. Physical Review A, 99(1), [013844].

https://doi.org/10.1103/PhysRevA.99.013844

\section{General rights}

Copyright and moral rights for the publications made accessible in the public portal are retained by the authors and/or other copyright owners and it is a condition of accessing publications that users recognise and abide by the legal requirements associated with these rights.

- Users may download and print one copy of any publication from the public portal for the purpose of private study or research.

- You may not further distribute the material or use it for any profit-making activity or commercial gain

- You may freely distribute the URL identifying the publication in the public portal

If you believe that this document breaches copyright please contact us providing details, and we will remove access to the work immediately and investigate your claim. 


\title{
Effects of higher-order dispersion on photon-pair generation by four-wave mixing
}

\author{
Jacob G. Koefoed, Jesper B. Christensen, Colin J. McKinstrie, and Karsten Rottwitt \\ Department of Photonics Engineering, Technical University of Denmark, 2800 Kongens Lyngby, Denmark
}

(Received 28 August 2018; published 23 January 2019)

\begin{abstract}
We present analytical results revealing the impact of higher-order dispersion on four-wave mixing in quantum applications. We derive fundamental relations between input and output modes in dispersive four-wave mixing processes and apply the theory to photon-pair generation. We analyze specific examples of four-wave-mixing configurations and find that, in some cases, spectral correlations are severely affected by dispersion while other cases are strongly unaffected. For the considered examples, we derive analytic conditions for dispersion cancellation, resulting in highly factorable two-photon states.
\end{abstract}

DOI: 10.1103/PhysRevA.99.013844

\section{INTRODUCTION}

Indistinguishable single photons are a key resource in quantum photonics applications such as interference-based linear optical quantum computing [1]. Traditionally, singlephoton sources for quantum optics experiments have been based on spontaneous parametric down-conversion (SPDC) in nonlinear crystals [2]. However, in recent years there has been an upsurge of interest in photon-pair generation in optical fibers [3-6] and integrated waveguides [7,8] through spontaneous four-wave mixing (SFWM). SFWM has the advantages of low coupling losses, high system stability, possibility of integration, and flexible schemes relying on nondegenerate pumps.

Both SFWM and SPDC sources probabilistically produce photons in pairs and the detection of one member of the pair heralds the presence of the other, which can subsequently be used in experiments $[9,10]$. The downside of the probabilistic production is compensated by having a trigger event and desirable photonic properties such as photons in the lowloss window of commercial fibers. Generally, the heralded photon is projected into an impure quantum state, unless the generated photon pair is completely spectrally unentangled $[11,12]$. This can be achieved through narrow spectral filtering on one or both photons [13], but at the cost of photon rate and heralding efficiency [14]. For this reason, there have been many attempts to engineer parametric sources to avoid such spectral correlations without the use of filters, relying instead on the careful design of the waveguide dispersion [15].

Nevertheless, even in sources that are engineered to produce uncorrelated photons the single-photon purity can degrade significantly due to a number of parasitic effects present in SPDC- or SFWM-based parametric sources. Research on the impact of such effects has mostly been focused on nonlinear phase modulation [16-18], dispersion fluctuations [19-21], and Raman scattering [22,23]. Notably, the impact of group-velocity dispersion (GVD) has been given very little attention; it is easily included in models for SPDC [24], but its

\footnotetext{
*jgko@fotonik.dtu.dk
}

effects on spectral correlations are negligible due to the short interaction lengths [25] and it has only been studied briefly in the context of SFWM [17].

In this work, we seek to characterize the impact of GVD on SFWM-based sources by developing analytical models for photon-pair generation where GVD is included and comparing them to numerical solutions for the two-photon amplitude. In addition, we consider the impact of pump chirp on photon purity in the presence of GVD. We focus on one of the most used schemes for generating pure heralded single photons, namely asymmetric group-velocity matching using a single pump, which has been realized in photonic crystal fibers [26,27]. We also examine a promising scheme based on complete collision between two pumps for which walk-off is induced by chromatic dispersion [28], waveguide birefringence [29], or higher-order fiber modes [21].

\section{DISPERSIVE FOUR-WAVE MIXING}

\section{A. Governing equations}

SFWM is enabled by third-order optical nonlinearities and consists of the annihilation of two pump photons to produce a signal and an idler photon under energy and momentum conservation. In the standard semiclassical approach the strong pumps are described classically, whereas the weak signal and idler fields are described by quantum Heisenberg annihilation operators $\hat{a}_{j}(z, \omega)$, with $j=s, r$ signifying the signal and idler, satisfying the equal-position commutation relations $\left[\hat{a}_{i}(z, \omega), \hat{a}_{j}^{\dagger}\left(z, \omega^{\prime}\right)\right]=\delta_{i j} \delta\left(\omega-\omega^{\prime}\right)$. In the Heisenberg picture (HP), the standard coupled-mode equations for the field-operator evolution in a waveguide are

$$
\begin{aligned}
\partial_{z} \hat{a}_{s}(z, \omega)= & i \beta_{s}(\omega) \hat{a}_{s}(z, \omega) \\
& +i \int d \omega^{\prime} \gamma_{p q}\left(z, \omega+\omega^{\prime}\right) \hat{a}_{r}^{\dagger}\left(z, \omega^{\prime}\right), \\
\partial_{z} \hat{a}_{r}(z, \omega)= & i \beta_{r}(\omega) \hat{a}_{r}(z, \omega) \\
& +i \int d \omega^{\prime} \gamma_{p q}\left(z, \omega+\omega^{\prime}\right) \hat{a}_{s}^{\dagger}\left(z, \omega^{\prime}\right),
\end{aligned}
$$


where the pump function, with the Fourier transform convention $f(\omega)=\int d t f(t) \exp (i \omega t)$, takes the form

$$
\gamma_{p q}(z, \omega)=\frac{\gamma}{2 \pi} \int d \omega^{\prime} A_{p}\left(z, \omega^{\prime}\right) A_{q}\left(z, \omega-\omega^{\prime}\right),
$$

where $A_{p}$ and $A_{q}$ are the pump fields, which are treated as classical and undepleted. The Kerr nonlinearity parameter $\gamma$ governs the interaction strength depending on the material properties as well as the central frequency, transverse spatial profile, and polarization of the light fields [30]. Due to the linearity of Eqs. (1), the field evolution through the waveguide can be conveniently expressed through input-output relations of the form

$$
\begin{aligned}
\hat{a}_{s}\left(\omega, z_{\text {out }}\right)= & \int d \omega^{\prime}\left[\mu_{s s}\left(\omega, \omega^{\prime}\right) \hat{a}_{s}\left(\omega^{\prime}, z_{\text {in }}\right)\right. \\
& \left.+v_{s r}\left(\omega, \omega^{\prime}\right) \hat{a}_{r}^{\dagger}\left(\omega^{\prime}, z_{\text {in }}\right)\right], \\
\hat{a}_{r}\left(\omega, z_{\text {out }}\right)= & \int d \omega^{\prime}\left[\mu_{r r}\left(\omega, \omega^{\prime}\right) \hat{a}_{r}\left(\omega^{\prime}, z_{\text {in }}\right)\right. \\
& \left.+v_{r s}\left(\omega, \omega^{\prime}\right) \hat{a}_{s}^{\dagger}\left(\omega^{\prime}, z_{\text {in }}\right)\right],
\end{aligned}
$$

where $\mu_{s s}$ and $\mu_{r r}$ are the frequency-domain self-Green functions and $v_{s r}$ and $v_{r s}$ are the frequency-domain cross-Green functions. Due to the symmetry between the signal and idler fields, we only consider the signal from this point. The self Green function $\mu_{s s}$ and cross Green function $v_{s r}$ can be found perturbatively (in the classical case, this corresponds to a zero-input idler), as

$$
\begin{gathered}
\hat{a}_{s}(\omega, l)=\exp \left[i \beta_{s}\left(\omega^{\prime}\right) l\right] \hat{a}_{s}(\omega, 0) \\
\hat{a}_{r}(\omega, l)=i \exp \left[i \beta_{r}(\omega) l\right] \int_{0}^{l} d z \int d \omega^{\prime} \gamma_{p q}\left(\omega+\omega^{\prime}, z\right) \\
\times \exp \left\{-i\left[\beta_{r}(\omega)+\beta_{s}\left(\omega^{\prime}\right)\right] z\right\} \hat{a}_{s}^{\dagger}\left(\omega^{\prime}, 0\right),
\end{gathered}
$$

where $l$ is the waveguide length. From these equations, the perturbative Green functions are seen to be

$$
\begin{aligned}
\mu_{s s}\left(\omega, \omega^{\prime}\right)= & \exp \left[i \beta_{s}(\omega) l\right] \delta\left(\omega-\omega^{\prime}\right), \\
v_{r s}\left(\omega, \omega^{\prime}\right)= & i \exp \left[i \beta_{r}(\omega) l\right] \int_{0}^{l} d z \gamma_{p q}\left(\omega+\omega^{\prime}, z\right) \\
& \times \exp \left\{-i\left[\beta_{r}(\omega)+\beta_{s}\left(\omega^{\prime}\right)\right] z\right\} .
\end{aligned}
$$

The signal-idler symmetry dictates that $v_{r s}$ is obtained from $v_{s r}$ by swapping $r \leftrightarrow s$ and $\omega \leftrightarrow \omega^{\prime}$. This combined with the symmetric form of the cross-Green function allows us to give nearly identical decompositions of the two cross functions as follows:

$$
\begin{aligned}
& v_{r s}\left(\omega, \omega^{\prime}\right)=\exp \left[i \beta_{r}(\omega) l\right] \sum_{n} v_{n} f_{r n}(\omega) f_{s n}\left(\omega^{\prime}\right), \\
& v_{s r}\left(\omega, \omega^{\prime}\right)=\exp \left[i \beta_{s}(\omega) l\right] \sum_{n} v_{n} f_{s n}(\omega) f_{r n}\left(\omega^{\prime}\right),
\end{aligned}
$$

where $f_{j n}, j=s, r$ is the $n$th Schmidt function (SF) for the signal and idler fields with Schmidt coefficient $v_{n}$. This shows that, although the input and output SFs depend on the nonlinear interaction throughout the medium, the output function $f_{j n}(\omega) \exp \left[i \beta_{j}(\omega) l\right]$, is simply a propagated and dispersed version of the input function, $f_{j n}(\omega)$. This is a generalization of the result obtained in [29]. Thus, if the Schmidt functions for a process are known, this gives a simple way to decompose an input field and propagate it. In the following section, we link this description to the Schrödinger picture (SP).

\section{B. Schrödinger picture}

The Heisenberg equations (1) can be generated from a Hamiltonian $\hat{H}(z)$, which governs spatial evolution such that $\partial_{z} \hat{a}_{s}=i\left[\hat{H}, \hat{a}_{s}\right]$ and similarly for the idler. Note that, while this operator is, strictly speaking, a momentum operator $[18,21,31]$, we shall refer to it as the Hamiltonian. To generate Eqs. (1), this operator must take the form

$$
\begin{aligned}
\hat{H}(z)= & i \int d \omega\left[\beta_{s}(\omega) \hat{a}_{s}^{\dagger}(\omega) \hat{a}_{s}\left(\omega^{\prime}\right)+\beta_{r}(\omega) \hat{a}_{r}^{\dagger}(\omega) \hat{a}_{r}(\omega)\right] \\
& +i \iint d \omega d \omega^{\prime} \gamma_{p q}\left(z, \omega+\omega^{\prime}\right) \hat{a}_{r}^{\dagger}(\omega) \hat{a}_{s}^{\dagger}\left(\omega^{\prime}\right)+\text { H.c. }
\end{aligned}
$$

To solve the evolution problem perturbatively, let $\hat{H}(z)=$ $\hat{H}_{0}+\hat{H}_{1}(z)$ where $\hat{H}_{0}$ describes dispersion [the first term in Eq. (8)] and $\hat{H}_{1}$ describes the SFWM interaction. Like the Hamiltonian, the state can also be expanded as $|\psi\rangle=\left|\psi_{0}\right\rangle+$ $\left|\psi_{1}\right\rangle$, which decomposes the evolution equation $\partial_{z}|\psi\rangle=$ $i \hat{H}|\psi\rangle$ into the two perturbative orders

$$
\begin{gathered}
\partial\left|\psi_{0}\right\rangle=i \hat{H}_{0}\left|\psi_{0}\right\rangle, \\
\partial\left|\psi_{1}\right\rangle=i \hat{H}_{0}\left|\psi_{1}\right\rangle+i \hat{H}_{1}\left|\psi_{0}\right\rangle .
\end{gathered}
$$

By introducing the unitary operator $U_{0}(z)=\exp \left(i \hat{H}_{0} z\right)$ generated by $\hat{H}_{0}$, these equations are easily solved to give

$$
\begin{gathered}
\left|\psi_{0}(l)\right\rangle=\hat{U}_{0}(l)\left|\psi_{0}(0)\right\rangle \\
\left|\psi_{1}(l)\right\rangle=i \int_{0}^{l} d z \hat{U}_{0}(l-z) \hat{H}_{1}(z)\left|\psi_{0}(z)\right\rangle .
\end{gathered}
$$

These equations have a simple interpretation. The zeroorder state evolves simply under $\hat{H}_{0}$, while the first-order state is produced by a series of creation events, through $\hat{H}_{1}(z)$, followed by propagation for the remaining waveguide length, through $\hat{U}_{0}(l-z)$. Rewriting the first-order expression slightly to

$$
\begin{aligned}
\left|\psi_{1}(l)\right\rangle & =i \hat{U}_{0}(l) \int_{0}^{l} d z \hat{U}_{0}^{\dagger}(z) \hat{H}_{1}(z) \hat{U}_{0}(z)\left|\psi_{0}(0)\right\rangle \\
& =i \hat{U}_{0}(l) \int_{0}^{l} d z \hat{H}_{\mathrm{int}}(z)\left|\psi_{0}(0)\right\rangle,
\end{aligned}
$$

reveals a transition to the interaction picture where the interaction Hamiltonian $\hat{H}_{\text {int }}(z)=\hat{U}_{0}^{\dagger}(z) \hat{H}_{1}(z) \hat{U}_{0}(z)$ is obtained from $\hat{H}_{1}$ by simply replacing the Schrödinger field operators by their corresponding $z$-dependent Heisenberg operators, which evolve according to $\partial_{z} \hat{a}_{j}=i\left[\hat{H}_{0}, \hat{a}_{j}\right]=i \beta_{j} \hat{a}_{j}$, so that $\hat{a}_{j}(z)=\hat{a}_{j} \exp \left(i \beta_{j} z\right)$, for $j=s, r$. We note that with the Hamiltonian in Eq. (8), the operator $\hat{U}_{0}(l)$ may be replaced by the complex number $\exp \left\{i\left[\beta_{s}\left(\omega^{\prime}\right)+\beta_{r}\left(\omega^{\prime \prime}\right)\right] l\right\}$ inside the 
double frequency integral in Eq. (8). By writing the twophoton part of the state as

$$
\left|\psi_{1}\right\rangle=\iint d \omega_{s} d \omega_{r} v\left(\omega_{s}, \omega_{r}\right) \hat{a}_{s}^{\dagger}\left(\omega_{s}\right) \hat{a}_{r}^{\dagger}\left(\omega_{r}\right)|\mathrm{vac}\rangle,
$$

where the field operators are Schrödinger operators $\hat{a}_{s}\left(\omega_{s}\right)=$ $\hat{a}_{s}\left(0, \omega_{s}\right)$, we see from Eqs. (13) and (8) [with $\hat{a}_{j}$ replaced by $\hat{a}_{j}(z)$ ] that the joint wave function $v\left(\omega_{s}, \omega_{r}\right)$ can be identified as

$$
\begin{aligned}
v\left(\omega_{s}, \omega_{r}\right)= & i \int_{0}^{l} d z \gamma_{p q}\left(z, \omega_{s}+\omega_{r}\right) \\
& \times \exp \left\{i\left[\beta_{s}\left(\omega_{s}\right)+\beta_{r}\left(\omega_{r}\right)\right][l-z]\right\} .
\end{aligned}
$$

With Eq. (3) as the definition of the frequency-domain Green functions, the two-photon amplitude $v$ and the Green function are related as [29]

$$
v\left(\omega_{s}, \omega_{r}\right)=\int d \omega \mu_{s s}\left(\omega_{s}, \omega\right) v_{r s}\left(\omega_{r}, \omega\right) .
$$

This relation shows that the Green functions from Eq. (6) in the HP and the two-photon amplitude Eq. (15) in the SP are consistent with the fact that the SP SFs correspond to the output HP SFs. For convenience, we let $v\left(\omega_{s}, \omega_{r}\right)=$ $\mathcal{A}\left(\omega_{s}, \omega_{r}\right) \exp \left\{i\left[\beta_{s}\left(\omega_{s}\right)+\beta_{r}\left(\omega_{r}\right)\right] l / 2\right\}$. This is a difference of an overall spectral phase from pure dispersion, which does not affect the spectral intensity or any two-photon correlations. We will refer to $\mathcal{A}\left(\omega_{s}, \omega_{r}\right)$ as the joint spectral amplitude (JSA). In addition, we, from this point, let the waveguide coordinate run from $-l / 2$ to $l / 2$ for convenience, i.e., we substitute $z \rightarrow z-l / 2$. Note that this means that an unchirped pump input is chirped at $z=0$ (the waveguide midpoint). Like the Green functions, the JSA can be Schmidt decomposed [12]

$$
\mathcal{A}\left(\omega_{s}, \omega_{r}\right)=\sum_{n} \lambda_{n} f_{s n}\left(\omega_{s}\right) f_{r n}\left(\omega_{r}\right)
$$

where $\lambda_{n}$ are real coefficients and $f_{s n}, f_{r n}$ are SP SFs, which are identical to the Heinsenberg functions in Eqs. (6) evaluated at the waveguide midpoint. From such a decomposition the spectral purity $P$ of a single photon heralded from the two-photon state is easily determined as

$$
P=\sum_{n} \lambda_{n}^{4} /\left(\sum_{n} \lambda_{n}^{2}\right)^{2}
$$

This expression can be found in the standard way from the trace of the square of the density operator after tracing out the idler photon and using the decomposition Eq. (17). In simple cases, analytic expression for the purity can be found, but one is often forced to calculate it numerically through a singular value decomposition and Eq. (18).

\section{Gaussian pumps and phase mismatch}

In this section we introduce the pumps explicitly and derive a general expression for the JSA with higher-order dispersion, which is used in subsequent sections. With the definitions in the previous section, we can from Eq. (15) write the
JSA as

$$
\begin{aligned}
\mathcal{A}\left(\omega_{s}, \omega_{r}\right)= & i \int_{-l / 2}^{l / 2} d z \gamma_{p q}\left(z, \omega_{s}+\omega_{r}\right) \\
& \times \exp \left\{-i\left[\beta_{s}\left(\omega_{s}\right)+\beta_{r}\left(\omega_{r}\right)\right] z\right\} \\
= & \frac{i \gamma}{2 \pi} \iint d z d \omega A_{p}\left(0, \omega_{\mathrm{a}}+\omega\right) A_{q}\left(0, \omega_{\mathrm{a}}-\omega\right) \\
& \times \exp [i \Delta \beta(\omega) z]
\end{aligned}
$$

where $\omega_{\mathrm{a}}=\left(\omega_{s}+\omega_{r}\right) / 2$ is the average frequency and we assume that the pump fields evolve under only dispersive effects, i.e., $\partial_{z} A_{j}=i \beta_{j}(\omega) A_{j}$. This is often reasonable with the relatively weak pump powers used in photon-pair-generation experiments. With these definitions, the wave-number mismatch is

$$
\begin{aligned}
\Delta \beta(\omega) & =\beta_{p}\left(\omega_{\mathrm{a}}+\omega\right)+\beta_{q}\left(\omega_{\mathrm{a}}-\omega\right)-\beta_{s}\left(\omega_{s}\right)-\beta_{r}\left(\omega_{r}\right) \\
& =\Delta \beta_{0}\left(\omega_{s}, \omega_{r}\right)+k\left(\omega_{s}, \omega_{r}\right) \omega+\frac{1}{2}\left(\beta_{2 p}+\beta_{2 q}\right) \omega^{2}
\end{aligned}
$$

where the last equality is an expansion in dispersion parameters up to second order around a point of perfect phase matching, i.e., the center of the two-photon state which we take as $\omega_{s}=\omega_{r}=0$. The expansion is given in terms of the expressions

$$
\begin{aligned}
\Delta \beta_{0}\left(\omega_{s}, \omega_{r}\right)= & \frac{1}{2}\left(\beta_{2 p}+\beta_{2 q}\right) \omega_{\mathrm{a}}^{2}-\beta_{1 s} \omega_{s}-\frac{1}{2} \beta_{2 s} \omega_{s}^{2} \\
& -\beta_{1 r} \omega_{r}-\frac{1}{2} \beta_{2 r} \omega_{r}^{2}, \\
k\left(\omega_{s}, \omega_{r}\right)= & \left(\beta_{2 p}-\beta_{2 q}\right) \omega_{\mathrm{a}}+\beta_{1 p}-\beta_{1 q},
\end{aligned}
$$

where $\beta_{n} j, j=s, r, p, q$ is the $n$th derivative of $\beta$ at the central frequency of field $j$. For convenience, we omit the arguments in future reference to these quantities. For the remainder of this paper, the pumps are assumed to be chirped Gaussians, which at the fiber midpoint take the forms

$$
\begin{aligned}
& A_{j}(0, t)=\sqrt{P_{j}} \exp \left(-\frac{\left(1+i C_{j}\right) t^{2}}{2 T_{j}^{2}}\right), \quad j=p, q, \\
& A_{j}(0, \omega)=A_{j 0} \exp \left(-\frac{\left(1-i C_{j}\right) \omega^{2}}{2 \sigma_{j}^{2}}\right), \quad j=p, q,
\end{aligned}
$$

with $A_{j 0}=\sigma_{j}^{-1} \sqrt{2 \pi P_{j}\left(1-i C_{j}\right)}$ and $\sigma_{j}=\sqrt{1+C_{j}^{2}} / T_{j}$ so that $\left|A_{j 0}\right|=\sqrt{2 \pi P_{j}} T_{j}$. Notice that in this notation, $C_{j}$ is the chirp at the waveguide midpoint since this is more convenient in the following analysis. In terms of the input parameters, they are

$$
\begin{gathered}
C_{p}=C_{\mathrm{in}}+\left(1+C_{\mathrm{in}}^{2}\right) \frac{\beta_{2 p} l}{2 T_{\mathrm{in}}^{2}}, \\
T_{p}=T_{\mathrm{in}}\left[\left(1+C_{\mathrm{in}} \frac{\beta_{2 p} l}{2 T_{\mathrm{in}}^{2}}\right)^{2}+\left(\frac{\beta_{2 p} l}{2 T_{\mathrm{in}}^{2}}\right)^{2}\right]^{1 / 2},
\end{gathered}
$$

with $C_{\text {in }}=0$ for unchirped input, and similarly for the pump $q$. Since the pump pulse durations change throughout the waveguide, but the spectral width $\sigma_{j}$ does not, it is often useful to express quantities in terms of $\sigma_{j}$ to avoid confusion. 
The integrals in Eq. (19) are difficult to evaluate analytically. However, results may be obtained for the most experimentally interesting cases. In the following sections we focus on one example of degenerate FWM (using a single pump) and one example of nondegenerate FWM (using two distinct pumps).

\section{DEGENERATE FWM}

\section{A. Approximate solution}

In this section we consider degenerate pumps, i.e., $A_{p}=$ $A_{q}$, which simplifies the phase mismatch in Eq. (20) to $\Delta \beta(\omega)=\Delta \beta_{0}+\beta_{2 p} \omega^{2}$. Carrying out the $z$ integral in Eq. (19) gives

$$
\begin{aligned}
\mathcal{A}\left(\omega_{s}, \omega_{r}\right)= & \frac{i \gamma l}{2 \pi} \int d \omega A_{p}\left(\omega_{\mathrm{a}}+\omega\right) A_{p}\left(\omega_{\mathrm{a}}-\omega\right) \\
& \times \operatorname{sinc}\left(\frac{\Delta \beta(\omega) l}{2}\right),
\end{aligned}
$$

where we take $A_{p}(\omega)$ to mean the field at the waveguide midpoint. This integral is commonly dealt with by neglecting the second-order contribution, and thus GVD, to $\Delta \beta(\omega)$. We include it by expanding the sinc function in Eq. (27) with argument $\Delta \beta(\omega) l / 2=\Delta \beta_{0} l / 2+\beta_{2 p} \omega^{2} l / 2$ with respect to $\beta_{2 p} \omega^{2} l / 2$

$$
\begin{aligned}
\operatorname{sinc}\left(\frac{\Delta \beta(\omega) l}{2}\right)= & \operatorname{sinc}\left(\frac{\Delta \beta_{0} l}{2}\right) \\
& +\frac{1}{2} \beta_{2 p} \omega^{2} l \operatorname{sinc}^{\prime}\left(\frac{\Delta \beta_{0} l}{2}\right) .
\end{aligned}
$$

This expansion is correct to order $\beta_{2 p}^{2} \omega^{4} l^{2}$, consistent with neglecting third- and higher-order dispersion. With this approximation, the integral in Eq. (27) is now a simple Gaussian integral that can be carried out to give

$$
\begin{aligned}
\mathcal{A}\left(\omega_{s}, \omega_{r}\right)= & \frac{i \sqrt{\pi} \gamma l P_{p}}{\sigma_{p}} \sqrt{1-i C_{p}} \exp \left(-\frac{\left(1-i C_{p}\right) \omega_{\mathrm{a}}^{2}}{\sigma_{p}^{2}}\right) \\
& \times\left[\operatorname{sinc}\left(\frac{\Delta \beta_{0} l}{2}\right)+\operatorname{sinc}^{\prime}\left(\frac{\Delta \beta_{0} l}{2}\right) \frac{\beta_{2 p} \sigma_{p}^{2} l}{4\left(1-i C_{p}\right)}\right] .
\end{aligned}
$$

Recombining this expression into a single sinc function, which is still consistent with the order of approximation, gives the final expression

$$
\begin{aligned}
\mathcal{A}\left(\omega_{s}, \omega_{r}\right)= & \frac{i \sqrt{\pi} \gamma l P_{p}}{\sigma_{p}} \sqrt{1-i C_{p}} \exp \left(-\frac{\left(1-i C_{p}\right) \omega_{\mathrm{a}}^{2}}{\sigma_{p}^{2}}\right) \\
& \times \operatorname{sinc}\left(\left[\Delta \beta_{0}+\frac{\beta_{2 p} \sigma_{p}^{2}}{2\left(1-i C_{p}\right)}\right] \frac{l}{2}\right) .
\end{aligned}
$$

Even though the corrective term in the sinc function does not depend on $\omega_{s}$ and $\omega_{r}$, it can influence the spectral correlations. However, simply ignoring this term and giving the phasematching function as $\operatorname{sinc}\left(\Delta \beta_{0} l / 2\right)$ (not truncated at order $\beta_{1}$ ) can give a good approximation in some cases, and is an approach taken by some authors [32]. We shall refer to this

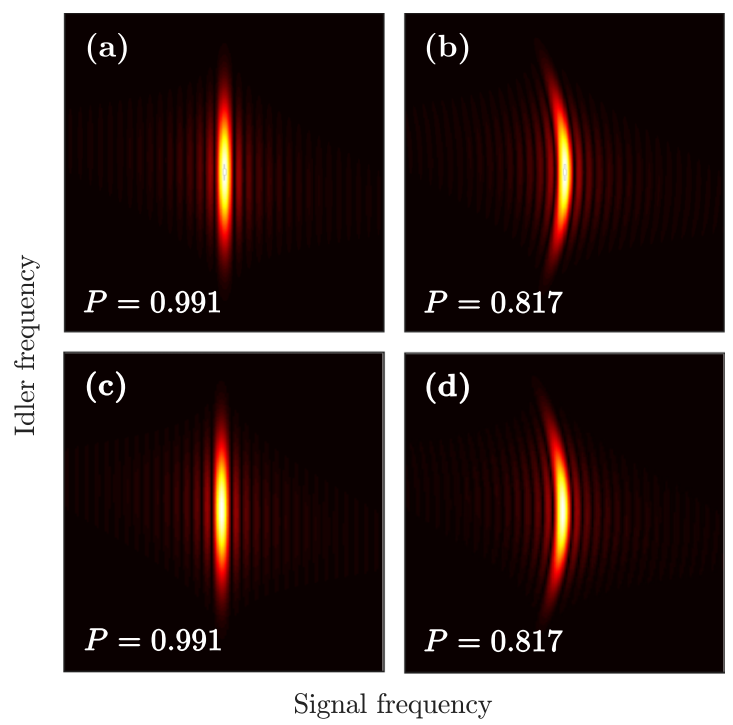

FIG. 1. Absolute value of analytical JSA (a) without and (b) with dispersion with $\beta_{1 s} l \sigma_{p}=100$, giving a high initial purity $\beta_{2 r} l \sigma_{p}^{2}=2$, $\beta_{2 p}=0$ and unchirped input. (c)-(d) show the corresponding numerical figures using a split-step algorithm reproducing the analytical amplitudes.

as a zero-order approximation. We note that in the case of SPDC, the JSA can always be given in this form without any approximations [11]. In the following we discuss a number of important consequences of the inclusion of GVD in Eq. (30).

\section{B. Asymmetric scheme for factorability}

Two degenerate schemes have been widely used to generate factorable two-photon states from which pure single photons can be heralded. In the symmetrically group-velocitymatched scheme, the signal and idler walk off symmetrically from the pump (one is faster, the other is slower), resulting in a central circular uncorrelated peak in the JSA, which can be subsequently filtered without significant losses. This scheme has been used both with SPDC [33-35] and SFWM [36-39]. Due to the symmetric spectral shape of the JSA, this scheme is less susceptible to GVD than the asymmetrically group-velocity-matched scheme in which either the signal or idler copropagates with the pump [12]. This scheme produces a JSA that is spectrally broad in the idler frequency and spectrally narrow in the signal frequency (or vice versa), see Fig. 1(a). Consequently, inclusion of GVD introduces a significant degree of curvature to the JSA, as shown in Fig. 1(b), calculated by Eq. (30). This increases spectral correlations, and for this choice of parameters which represents a large, but not unrealistic, amount of dispersion, reduces the heralded single-photon purity from a near-unit value to $81.8 \%$, calculated through Eq. (18). The corresponding plots from a numerical split-step scheme $[30,40]$ are shown in Figs. 1(c) and 1(d) and are identical to the analytical solutions. The split-step scheme is based on a nonlinear-Schrödingerlike propagation equation for the JSA, whereby any desired effects, such as higher-order dispersion, can be included. The impact of GVD on the Schmidt decomposition of the 

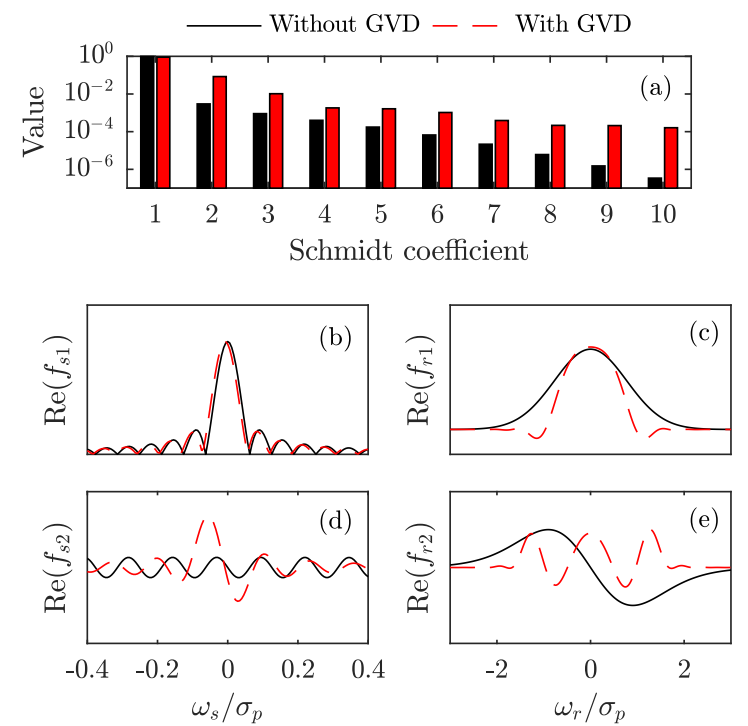

FIG. 2. (a) Absolute square of the Schmidt coefficients and (b)-(d) signal and idler Schmidt functions with and without dispersion for the parameters used in Fig. 1, showing significant degradation in purity and distortion of the Schmidt functions.

state is shown in Fig. 2, with black indicating no GVD and red indicating that GVD is present. As expected from the increased correlation, the Schmidt coefficients, apart from the first, increase in value when GVD is introduced. The first signal SF $f_{s 1}$ is nearly unchanged except for a slight shift. The idler SF $f_{r 1}$ is, however, distorted to account for the more narrow spectral distribution of the central part of the state due to its curvature. The second SFs, which account for the primary part of departure from a perfectly factorable state, are significantly different with and without dispersion. In the case of no dispersion, these functions describe the correlation remaining in the sinc ripples, inherent to the asymmetric scheme, due to the finite interaction length. However, when GVD is introduced, the resulting curvature becomes the dominant departure from perfect factorability, and the SFs $f_{s 2}$ and $f_{r 2}$ partially produces the curvature in the GVD-distorted JSA.

The origin of the correlations introduced by GVD are evident from the correction to the sinc argument compared to the case without GVD:

$$
\frac{1}{4} \beta_{2 p}\left(\omega_{s}+\omega_{r}\right)^{2}-\frac{1}{2} \beta_{2 s} \omega_{s}^{2}-\frac{1}{2} \beta_{2 r} \omega_{r}^{2}+\frac{\beta_{2 p} \sigma_{p}^{2}}{4\left(1-i C_{p}\right)}
$$

Due to the asymmetric nature of the JSA, GVD-induced correlations arise dominantly from terms proportional to $\omega_{r}^{2}$, i.e., $(1 / 2)\left(\beta_{2 p} / 2-\beta_{2 r}\right) \omega_{r}^{2}$. Thus, the impact of GVD is determined by an effective dispersion parameter $\beta_{2 p} / 2-\beta_{2 r}$. In particular, when $\beta_{2 p}=2 \beta_{2 r}$ we expect a large degree of dispersion cancellation. This is confirmed by Fig. 3, which shows the heralded single-photon purity as a function of idler GVD for different degrees of pump GVD. This figure additionally confirms that the behavior is nearly completely determined by the value of $\left|\beta_{2 p} / 2-\beta_{2 r}\right|$ from the pairwise similarity of the plotted lines. For the figure we chose $\beta_{2 s}=0$, but for the

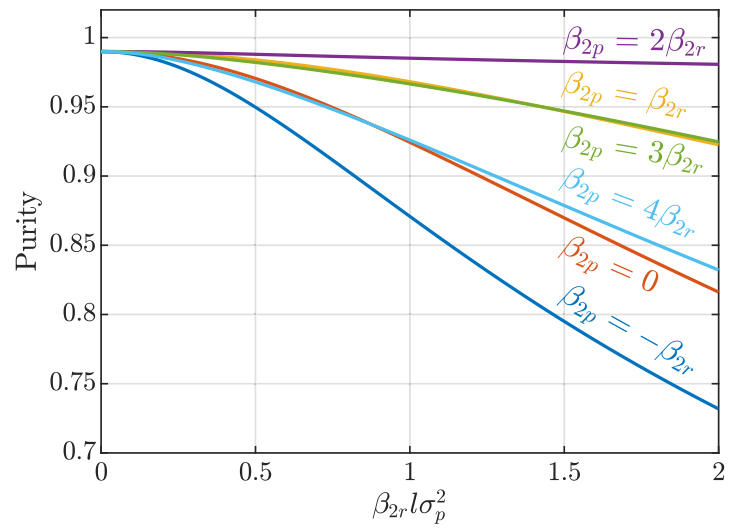

FIG. 3. Heralded single-photon purity versus idler dispersion for varying amount of pump dispersion (indicated adjacent to the lines) with $\beta_{1 s} l \sigma_{p}=100$ and unchirped input, i.e., $\sigma_{p}=1 / T_{\text {in }}$, showing the purity-degrading effects on the asymmetric scheme.

degree of asymmetry considered here, this parameter has no influence on the purity. The figure illustrates that, while the purity always decreases with increasing idler GVD, the impact is significantly smaller when $\beta_{2 p} \approx 2 \beta_{2 r}$, as predicted. This shows that nearly perfect dispersion cancellation is possible. However, in real fibers designed for the asymmetric scheme, the GVD often has opposite sign for the pump and idler, enhancing the negative effect instead of inhibiting it [17,26,32].

Perhaps the most important consequence of GVD is the limit it imposes on the maximum achievable purity for the asymmetric scheme. Figure 4 shows the purity versus fiber length for varying pump pulse duration, with and without GVD for realistic fiber parameters in a photonic crystal fiber. Without GVD, the purity increases monotonically and goes asymptotically to unity as the fiber length is increased. However, when GVD is accounted for, the purity increases to some maximum value and then starts to decrease due to GVD, resulting in an optimal fiber length for any given

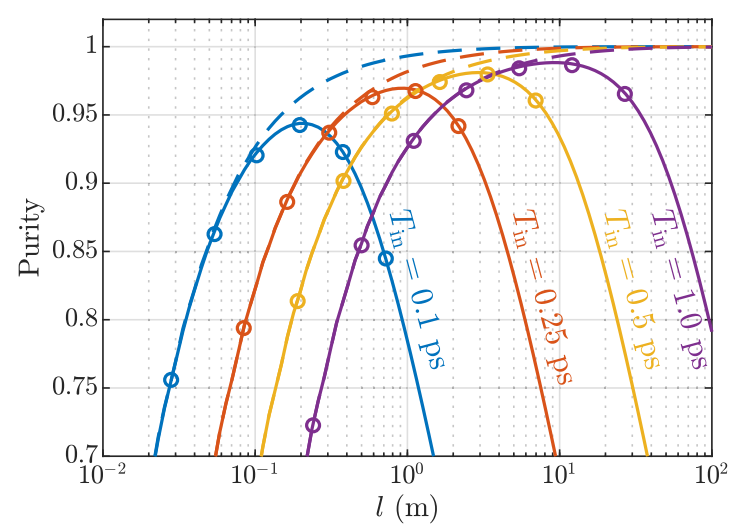

FIG. 4. Purity versus fiber length for different pulse durations (indicated next to the lines) and unchirped input. Dashed lines indicate the case with no GVD and circles are results from a numerical split-step routine. The parameters used are $\beta_{1 s}=1.14 \times$ $10^{-11} \mathrm{~m}^{-1} \mathrm{~s}, \beta_{2 p}=2.1 \times 10^{-26} \mathrm{~m}^{-1} \mathrm{~s}^{2}, \beta_{2 r}=-1.3 \times 10^{-26} \mathrm{~m}^{-1} \mathrm{~s}^{2}$, $\beta_{2 s}=3.6 \times 10^{-26} \mathrm{~m}^{-1} \mathrm{~s}^{2}$ [17]. GVD prevents a monotonic increase in purity with fiber length. 


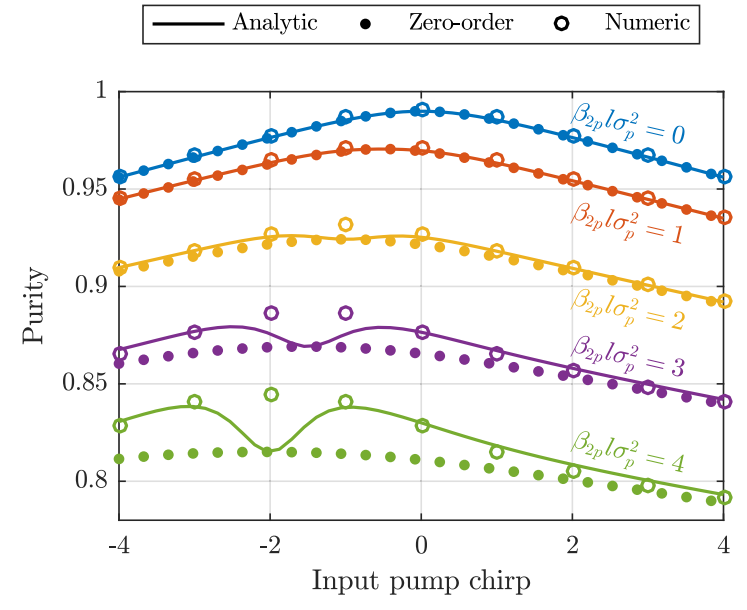

FIG. 5. Purity versus input pump chirp for varying amounts of GVD (indicated next to the lines) for our analytical solution (solid lines), the zero-order approximation without the correction to $\Delta \beta_{0}$ (dotted lines), and numerical values from the split-step solver (circles), showing good agreement even for strong dispersion for most chirps. The purity exhibits a weak dependence on pump chirp, even for strong dispersion.

pulse duration. Furthermore, for a given amount of GVD, this maximum value decreases with decreasing pump pulse duration, due to the increased effect of GVD for shorter pulses. This has consequences for the design of experiments using this scheme which is often constrained by available fiber length, e.g., a few meters in the case of photonic crystal fibers [17] due to fiber inhomogeneities. Our results show that in a dispersive fiber there is an optimal pump pulse duration, depending on the maximum available fiber length. The results are confirmed with a split-step routine (circles in Fig. 4). The excellent agreement indicates that the expansion we used for the sinc function gives a very good approximation.

Finally, we investigate the effect of pump chirp on the quantum state. The temporal pump chirp included in our model has two consequences for the generated state. The first is a spectral broadening, which has an obvious effect on the impact of dispersion, as shown in Fig. 4. The second, which can be isolated by fixing the pump spectral width $\sigma_{p}$ to varying constant values, is shown in Fig. 5, with solid lines showing our analytical model, dotted lines a naive model without the corrective term in the sinc from the Taylor expansion, and circles from the numerical split-step scheme, all for varying GVD, measured by $\beta_{2 p} l \sigma_{p}^{2}$. We first note that the analytical solution presented here performs much better than the simple analytical approximation, but shows some deviation when the pump is unchirped at the waveguide midpoint. At these points the analytical solution nearly coincides with the zero-order solution, indicating that higher-order terms are needed to capture the effect of GVD. Importantly, our analytical solution shows good agreement with the numerical solution for unchirped inputs, which is common in experimental situations. The figure shows that the optimal purity is always obtained for a pump pulse that is unchirped at the waveguide midpoint. However, the effect is modest even for large amounts of dispersion.

\section{NONDEGENERATE FWM}

We now turn our attention to the case in which the pumps are nondegenerate (in, e.g., frequency, polarization, or spatial mode) and denote them by $A_{p}$ and $A_{q}$. In general, these fields travel with different group velocities in the waveguide, making analytic progress difficult when GVD is included, although it is tractable when GVD is neglected [15]. However, simple analytical expressions can be found when the two pump fields are allowed to pass completely through each other. This can enable perfectly factorable two-photon states and is therefore experimentally interesting $[21,28,29]$.

Recall the JSA given in Eq. (19)

$$
\begin{aligned}
\mathcal{A}\left(\omega_{s}, \omega_{r}\right)= & i \gamma \int d \omega A_{p}\left(\omega_{\mathrm{a}}+\omega\right) A_{q}\left(\omega_{\mathrm{a}}-\omega\right) \\
& \times \frac{1}{2 \pi} \int_{-l / 2}^{l / 2} d z \exp [i \Delta \beta(\omega) z] .
\end{aligned}
$$

A complete collision occurs in the limit $l \rightarrow \infty$, and is approximately achieved if the pump-pump walk-off is much larger than their duration, i.e., $\left|\beta_{1 p}-\beta_{1 q}\right| l \gg \sqrt{T_{p}^{2}+T_{q}^{2}}$, or, equivalently, $l \gg l_{\text {col }}$ for the collision length

$$
l_{\mathrm{col}}=\frac{\sqrt{T_{p}^{2}+T_{q}^{2}}}{\left|\beta_{1 p}-\beta_{1 q}\right|} .
$$

In the complete-collision limit $l \rightarrow \infty$, the $z$ integral becomes a delta function with contributions only for $\Delta \beta(\omega)=0$, that is,

$$
\mathcal{A}\left(\omega_{s}, \omega_{r}\right)=i \gamma \int d \omega A_{p}\left(\omega_{\mathrm{a}}+\omega\right) A_{q}\left(\omega_{\mathrm{a}}-\omega\right) \delta[\Delta \beta(\omega)] .
$$

With higher-order dispersion, the equation $\Delta \beta(\omega)=0$ may have multiple solutions. We focus on the particular solution $\omega_{0}$ which has the property that $\omega_{0}=0$ when $\omega_{s}=\omega_{r}=0$, thus giving a JSA centered around $\omega_{s}=\omega_{r}=0$. Other solution have frequency detunings that are much greater than the pump spectral width and would in an experimental situation be removed with spectral filtering. In the edge case of $\Delta \beta^{\prime}\left(\omega_{0}\right)=$ 0 the integral is undefined and it is zero if the solution $\omega_{0}$ does not exist. Thus, by defining

$$
F\left(\omega_{s}, \omega_{r}\right)= \begin{cases}\left|\Delta \beta^{\prime}\left(\omega_{0}\right)\right|^{-1}, & \omega_{0} \text { exists and } \Delta \beta^{\prime}\left(\omega_{0}\right) \neq 0 \\ 0, & \text { otherwise }\end{cases}
$$

we may write the integral simply as

$$
\mathcal{A}\left(\omega_{s}, \omega_{r}\right)=i \gamma F\left(\omega_{s}, \omega_{r}\right) A_{p}\left(\omega_{\mathrm{a}}+\omega_{0}\right) A_{q}\left(\omega_{\mathrm{a}}-\omega_{0}\right) .
$$

In the case where dispersion up to second order is included, we can obtain explicit expressions for $\omega_{0}$ and $\Delta \beta^{\prime}\left(\omega_{0}\right)$ :

$$
\begin{array}{r}
\Delta \beta(\omega)=\Delta \beta_{0}+k \omega+\frac{1}{2}\left(\beta_{2 p}+\beta_{2 q}\right) \omega^{2}, \\
\omega_{0}\left(\omega_{s}, \omega_{r}\right)=\frac{-k+\sqrt{k^{2}-2\left(\beta_{2 p}+\beta_{2 q}\right) \Delta \beta_{0}}}{\beta_{2 p}+\beta_{2 q}}, \\
\Delta \beta^{\prime}\left(\omega_{0}\right)=\sqrt{k^{2}-2\left(\beta_{2 p}+\beta_{2 q}\right) \Delta \beta_{0}},
\end{array}
$$




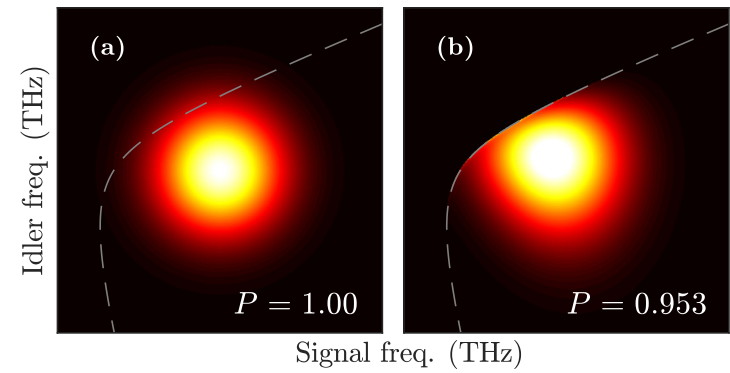

FIG. 6. JSA (a) with and (b) without dispersion. The dashed line indicates cutoff from the Heaviside function in Eq. (40). We here assume pairwise group-velocity matching $\beta_{1 p}=\beta_{1 s}$ and $\beta_{1 q}=\beta_{1 r}$ and the parameters $\beta_{2} l / T_{p}^{2}=4, T_{p}=T_{q}, \beta_{2 p}=\beta_{2}, \beta_{2 q}=\beta_{2 s}=$ $0.9 \beta_{2}$, and $\beta_{2 r}=0.2 \beta_{2}$ with pumps unchirped at the collision point. Even strong GVD only perturbs the state weakly.

where $\Delta \beta_{0}$ and $k$ are as defined in Eqs. (21) and (22). Additionally, the requirement of existence of $\omega_{0}$ is simple for this quadratic frequency dependence, yielding the more transparent result

$$
\mathcal{A}\left(\omega_{s}, \omega_{r}\right)=i \gamma \frac{\Theta\left[\Delta \beta_{0}^{\prime}\left(\omega_{0}\right)\right]}{\Delta \beta_{0}^{\prime}\left(\omega_{0}\right)} A_{p}\left(\omega_{\mathrm{a}}+\omega_{0}\right) A_{q}\left(\omega_{\mathrm{a}}-\omega_{0}\right),
$$

where $\Theta$ is a Heaviside function. For the remainder of this section we focus on Gaussian pumps with equal duration $T_{p}=T_{q}$. In this case, the effect of GVD on the otherwise uncorrelated state in Fig. 6(a) is seen in Fig. 6(b). The inclusion of GVD introduces a spectral cutoff for the state through the Heaviside function, indicated by the dashed line. This forces the state away from its uncorrelated circular shape and introduces correlations, thus reducing purity from unity to $95.3 \%$. However, an extremely large amount of dispersion is necessary to achieve this effect $\left(\beta_{2} l \sigma_{p}^{2} \approx 4\right)$, compared to, e.g., the asymmetric case analyzed in the previous section. Note that the singularity at the cutoff is integrable due to its square-root dependence on frequency, but may cause some numerical problems unless care is taken. Similarly to the asymmetric case, we illustrate the properties of the Schmidt decomposition with and without dispersion in Fig. 7. Unlike for the asymmetric scheme, the first SFs are only very slightly distorted from their initial Gaussian shape. However, there are now many active Schmidt modes, that combine to create the distortion and cutoff shown in Fig. 6.

To quantify the impact of GVD, we perturbatively expand $\omega_{0}=\omega_{1}+\delta \omega$. To lowest order

$$
\omega_{1}=\frac{\beta_{1 s} \omega_{s}+\beta_{1 r} \omega_{r}}{\beta_{1 p}-\beta_{1 q}} .
$$

This corresponds to a complete neglect of GVD and is in agreement with previous results [28,29]. The correction is found to be

$$
\delta \omega=\frac{\beta_{2 p}\left(\omega_{\mathrm{a}}+\omega_{1}\right)^{2}+\beta_{2 q}\left(\omega_{\mathrm{a}}-\omega_{1}\right)^{2}-\beta_{2 s} \omega_{s}^{2}-\beta_{2 r} \omega_{r}^{2}}{2\left(\beta_{1 p}-\beta_{1 q}\right)},
$$

which has a complicated dependence on the dispersion parameters. Near complete dispersion cancellation is obtained when
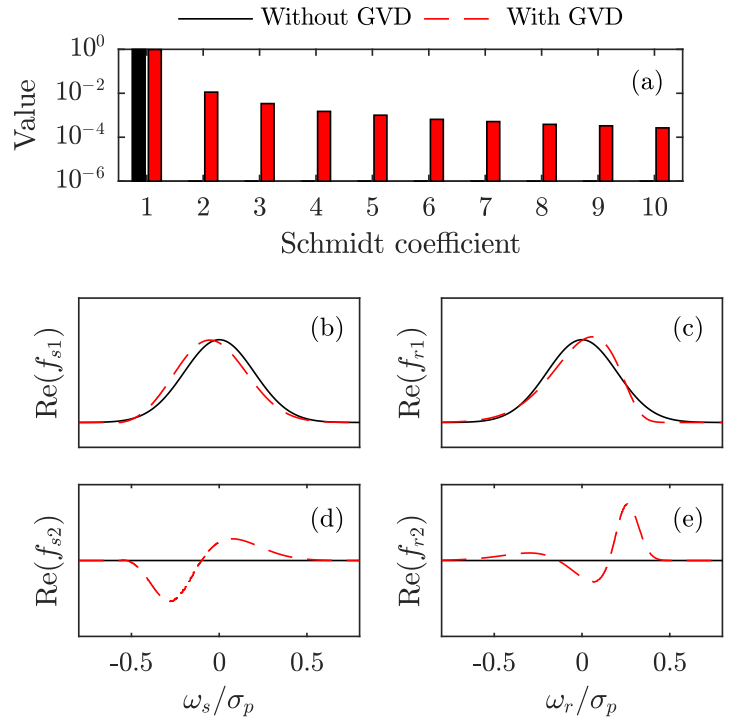

FIG. 7. (a) Absolute square of the Schmidt coefficients and (b)-(d) signal and idler Schmidt functions with and without dispersion for parameters identical to Fig. 6. GVD introduces additional Schmidt modes, but the smallness of the coefficients result in only weak perturbations.

$\delta \omega=0$ for all $\omega_{s}$ and $\omega_{r}$. This requirement leads to the three equations

$$
\begin{aligned}
\beta_{s q} \beta_{2 p}-\beta_{s p} \beta_{2 q} & =\beta_{p q} \beta_{2 s}, \\
\beta_{r q} \beta_{2 p}-\beta_{r p} \beta_{2 q} & =\beta_{p q} \beta_{2 r}, \\
\beta_{r q} \beta_{s q} \beta_{2 p}+\beta_{r p} \beta_{s p} \beta_{2 q} & =0,
\end{aligned}
$$

where $\beta_{i j}$ is the group-slowness difference between field $i$ and $j$. This set of equations determines three of the four GVD parameters, which are all proportional to the remaining one. In the simplest, and arguably the most experimentally interesting case of pairwise group-velocity matching $\left(\beta_{1 p} \approx \beta_{1 s}\right.$ and $\beta_{1 q} \approx \beta_{1 r}$ ) these equations reduce to $\beta_{2 p}=\beta_{2 s}$ and $\beta_{2 q}=$ $\beta_{2 r}$, i.e., the effects of dispersion cancel if the copropagating fields experience similar GVD. This is more likely to be the case if the fields are spectrally close to each other. Conversely, if all the fields experience nearly the same $\operatorname{GVD}\left(\beta_{2 s} \approx \beta_{2 r} \approx\right.$ $\beta_{2 p} \approx \beta_{2 q}$ ), these equations reduce to the single equation $\beta_{r q} \beta_{s q}+\beta_{r p} \beta_{s p}=0$, which is exactly the condition for having a factorable JSA with two identical Gaussian pumps [29]. Thus, if a collision-based scheme, using spectrally identical Gaussian pumps, is designed to produce a factorable JSA, the scheme is automatically robust to the effects of GVD. Unlike for the asymmetric scheme, implementations of this scheme can easily partially fulfill the requirements for dispersion cancellation using two modes that experience approximately equal GVD [23,29].

The effect of dispersion cancellation is confirmed by Fig. 8, which shows the purity versus the amount of GVD for four different combinations of dispersion parameters. As noted, the case where all four GVD parameters are nearly identical (blue curves with stars) is extremely robust to dispersion, with purities exceeding 0.999 for even large amounts of dispersion. The little remaining correlation is contained in the remaining 


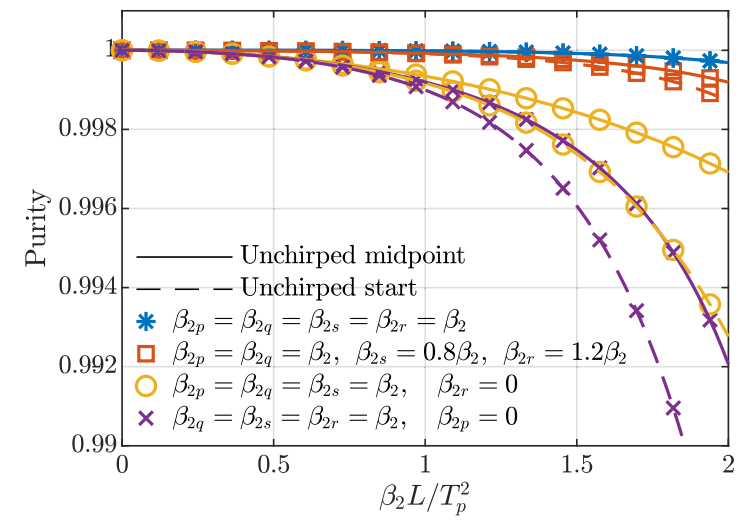

FIG. 8. Purity versus GVD for different relative values of the GVD parameters. The solid lines show the case where the pumps are unchirped at the waveguide midpoint while the dashed lines indicate unchirped pumps at the input with $l=10 l_{\text {col }}$. The overlayed symbols indicate four different cases for the dispersion parameters. In all cases the purity is nearly unaffected by the introduction of GVD.

factors in Eq. (40), indicating that the Gaussians contain the deciding part of the correlation. Introducing a slight asymmetry (red curve with squares) barely degrades performance. If one of the GVD parameters are much smaller than the others (yellow curves with circles and purple curves with crosses), which is possible with this configuration [21], the effect is again larger, especially if the pump dispersion is low, but the overall effect is still very small. This shows that the scheme is generally very robust and GVD is unlikely to be a concern in any real system. This is in contrast to the asymmetric scheme for pure photon generation described earlier, where realistic amounts of GVD could cause severe degradations in purity. The figure also shows the impact of pump chirp. We see that as for the asymmetric scheme, prechirping the pumps so they are unchirped at the collision point improves the heralded purity. However, the improvement is very modest and would likely be insignificant compared to other sources of degradation such as waveguide inhomogeneity, nonlinear phase modulation, or incomplete pump collision in real systems.

\section{CONCLUSION}

In this paper, we make a detailed study of the effects of dispersion on spontaneous four-wave mixing in the quantum regime. In the Heisenberg picture, we derive formulas for the Green functions and describe the general properties of their Schmidt decompositions. Although the Schmidt coefficients and the Schmidt functions are determined by the nonlinear interaction between the pump, signal, and idler, the output Schmidt functions are just the delayed and dispersed images of the input Schmidt functions. In the interaction picture, we derive an expression for the two-photon amplitude and describe the properties of its Schmidt decomposition. In particular, we show that its Schmidt functions are the output Heisenberg Schmidt functions. These results are valid for degenerate and nondegenerate four-wave mixing, arbitrary dispersion, and arbitrary pump pulses.

These results were then applied to two experimentally interesting examples, namely photon-pair generation using the asymmetrically group-velocity-matched scheme $\left(\beta_{1 p}=\right.$ $\beta_{1 s} \neq \beta_{1 r}$ ) and a complete pump-pump collision scheme $\left(\beta_{1 p} \neq \beta_{1 q}\right)$, both of which are useful for generating heralded single photons in spectrally pure quantum states.

We find an approximate expression for the two-photon amplitude in the asymmetric scheme and show that, although near-perfect dispersion cancellation is possible, photon-pair generation is negatively affected in waveguides with realistic dispersion. Notably, the introduction of higher-order dispersion yields an optimal pump pulse duration for a given length of waveguide.

Lastly, we find an exact analytical solution for the collision scheme and use it to show that this scheme is generally extremely resistant to the negative effects of dispersion. Also in this case we derive analytical conditions for dispersion cancellation and argue that in real implementations partial dispersion cancellation is realistic.

\section{ACKNOWLEDGMENT}

This work was supported by the Danish Council for Independent Research (DFF) (4184-00433).
[1] E. Knill, R. Laflamme, and G. J. Milburn, Nature 409, 46 (2001).

[2] P. G. Kwiat, K. Mattle, H. Weinfurter, A. Zeilinger, A. V. Sergienko, and Y. Shih, Phys. Rev. Lett. 75, 4337 (1995).

[3] M. Fiorentino, P. L. Voss, J. E. Sharping, and P. Kumar, IEEE Phot. Tech. Lett. 14, 983 (2002).

[4] X. Li, P. L. Voss, J. E. Sharping, and P. Kumar, Phys. Rev. Lett. 94, 053601 (2005).

[5] J. E. Sharping, J. Chen, X. Li, P. Kumar, and R. S. Windeler, Opt. Express 12, 3086 (2004).

[6] J. Rarity, J. Fulconis, J. Duligall, W. Wadsworth, and P. S. J. Russell, Opt. Express 13, 534 (2005).

[7] J. E. Sharping, K. F. Lee, M. A. Foster, A. C. Turner, B. S. Schmidt, M. Lipson, A. L. Gaeta, and P. Kumar, Opt. Express 14, 12388 (2006).

[8] C. Xiong et al., Opt. Lett. 36, 3413 (2011).
[9] S. Fasel, O. Alibart, S. Tanzilli, P. Baldi, A. Beveratos, N. Gisin, and H. Zbinden, New J. Phys. 6, 163 (2004).

[10] A. R. McMillan, J. Fulconis, M. Halder, C. Xiong, J. Rarity, and W. J. Wadsworth, Opt. Express 17, 6156 (2009).

[11] W. P. Grice and I. A. Walmsley, Phys. Rev. A 56, 1627 (1997).

[12] A. B. U'Ren, C. Silberhorn, R. Erdmann, K. Banaszek, W. P. Grice, I. A. Walmsley, and M. G. Raymer, Laser Phys. 15, 146 (2005).

[13] A. M. Brańczyk, T. Ralph, W. Helwig, and C. Silberhorn, New J. Phys. 12, 063001 (2010).

[14] A. Christ and C. Silberhorn, Phys. Rev. A 85, 023829 (2012).

[15] K. Garay-Palmett, H. McGuinness, O. Cohen, J. Lundeen, R. Rangel-Rojo, A. U'ren, M. Raymer, C. McKinstrie, S. Radic, and I. Walmsley, Opt. Express 15, 14870 (2007).

[16] L. Mejling, D. Cargill, C. McKinstrie, K. Rottwitt, and R. Moore, Opt. Express 20, 27454 (2012). 
[17] B. Bell, A. McMillan, W. McCutcheon, and J. Rarity, Phys. Rev. A 92, 053849 (2015).

[18] G. F. Sinclair and M. G. Thompson, Phys. Rev. A 94, 063855 (2016).

[19] L. Cui, X. Li, and N. Zhao, Phys. Rev. A 85, 023825 (2012).

[20] R. J. Francis-Jones and P. J. Mosley, Opt. Express 24, 24836 (2016).

[21] J. G. Koefoed, S. M. Friis, J. B. Christensen, and K. Rottwitt, Opt. Express 25, 20835 (2017).

[22] Q. Lin, F. Yaman, and G. P. Agrawal, Phys. Rev. A 75, 023803 (2007).

[23] J. G. Koefoed, J. B. Christensen, and K. Rottwitt, Phys. Rev. A 95, 043842 (2017).

[24] W. P. Grice, A. B. U'Ren, and I. A. Walmsley, Phys. Rev. A 64, 063815 (2001).

[25] Y. Jeronimo-Moreno and A. B. U'Ren, Phys. Rev. A 79, 033839 (2009).

[26] O. Cohen, J. S. Lundeen, B. J. Smith, G. Puentes, P. J. Mosley, and I. A. Walmsley, Phys. Rev. Lett. 102, 123603 (2009).

[27] M. Halder, J. Fulconis, B. Cemlyn, A. Clark, C. Xiong, W. J. Wadsworth, and J. G. Rarity, Opt. Express 17, 4670 (2009).

[28] B. Fang, O. Cohen, J. B. Moreno, and V. O. Lorenz, Opt. Express 21, 2707 (2013).
[29] J. B. Christensen, C. J. McKinstrie, and K. Rottwitt, Phys. Rev. A 94, 013819 (2016).

[30] G. P. Agrawal, Nonlinear Fiber Optics, 4th ed., (Elsevier, Amsterdam, 2006).

[31] I. Abram and E. Cohen, Phys. Rev. A 44, 500 (1991).

[32] R. J. Francis-Jones, R. A. Hoggarth, and P. J. Mosley, Optica 3, 1270 (2016).

[33] O. Kuzucu, F. N. C. Wong, S. Kurimura, and S. Tovstonog, Phys. Rev. Lett. 101, 153602 (2008).

[34] A. Eckstein, A. Christ, P. J. Mosley, and C. Silberhorn, Phys. Rev. Lett. 106, 013603 (2011).

[35] P. G. Evans, R. S. Bennink, W. P. Grice, T. S. Humble, and J. Schaake, Phys. Rev. Lett. 105, 253601 (2010).

[36] B. J. Smith, P. Mahou, O. Cohen, J. Lundeen, and I. Walmsley, Opt. express 17, 23589 (2009).

[37] C. Söller, O. Cohen, B. J. Smith, I. A. Walmsley, and C. Silberhorn, Phys. Rev. A 83, 031806 (2011).

[38] J. B. Spring et al., Opt. Express 21, 13522 (2013).

[39] J. B. Spring et al., Optica 4, 90 (2017).

[40] J. G. Koefoed, J. B. Christensen, and K. Rottwitt, in European Quantum Electronics Conference (Optical Society of America, Washington, D.C., 2017), p. EA_P_23. 